\title{
«Leutum meum bonum»: i silenzi di Petrarca sulla musica
}

\section{Chiara Cappuccio}

Universitat Autònoma de Barcelona

\section{Luca Zuliani}

Università di Padova

\begin{abstract}
Larticolo nasce da studi più generali riguardanti, per Chiara Cappuccio, la relazione tra poesia e musica nella lirica romanza delle origini, e per Luca Zuliani lo studio dei rapporti tra sintassi e metro nella poesia italiana fino al '500. La prima parte, Prassi esecutiva e riflessione teorica: su alcuni indizi musicali in Petrarca, vuole riunire in un disegno compiuto i frammenti del discorso di Petrarca sulla musica, rintracciati innanzitutto nelle sue opere latine. La seconda, La musica e la poesia volgare di Petrarca, cerca invece di definire, nell'ambito della questione dei rapporti fra musica e poesia nel Medioevo italiano, quali legami potessero ancora esistere fra la prassi musicale dell'epoca ed i componimenti volgari di Petrarca.
\end{abstract}

Parole chiave: Petrarca, musica, Ars nova, monodia.

\begin{abstract}
This essay results from broader researches of both the authors: Chiara Cappuccio is working on the relations between poetry and music in the Romance lyric poetry of the Middle Ages, Luca Zuliani is working on the connections between syntax and metre in the Italian poetry, from the beginnings to the $16^{\text {th }}$ century. The first part of the essay, Prassi esecutiva e riflessione teorica: su alcuni indizi musicali in Petrarca, collects and classifies the remarks made by Petrarch about music (which are found, mostly, in his Latin works), and tries to organize them in a coherent theory. The second part, La musica e la poesia volgare in Petrarca, examines the connection between music and poetry in the Italian Middle Ages and tries to find out which ties could still bind Petrarch's Tuscan poems to the contemporary musical praxis.
\end{abstract}

Key words: Petrarch, music, Ars nova, monody. 
Note al margine di più generali riflessioni riguardanti, da un lato, la relazione tra poesia e musica nella lirica romanza delle origini, dall'altro quella tra sintassi e metro nella poesia italiana fino al ' 500 , le considerazioni che seguono testimoniano la loro natura di lavori ancora in corso, appunti sparsi nati dall'analisi degli indizi "musicali» disseminati nell'opera di Petrarca. Organizzate intorno ad alcune suggestioni testuali presenti soprattutto nell'opera latina dell'autore, tali osservazioni costituiscono un cantiere aperto agli spunti critici su una figura apparentemente già così lontana dalla musica -intesa come prassi esecutiva e come sapere teorico- ma, allo stesso tempo, così determinante nel successivo sviluppo del linguaggio madrigalistico-polifonico.

Lasciando volutamente da parte quest'ultimo aspetto, focalizzeremo l'analisi dei luoghi musicali presenti all'interno dell'intera produzione dell'autore, da un lato verso una possibilità archeologica di ricostruzione di alcune delle prassi esecutive del testo lirico ancora in vita nel ' 300 , dall'altro verso una ricognizione più generale sui frammenti di un eventuale discorso di Petrarca sulla musica. ${ }^{1}$

1. Gli autori ringraziano per i preziosi aiuti e suggerimenti Gabriele Baldassari, Guido Capovilla, Francesco Facchin, Pier Vincenzo Mengaldo, Zeno Verlato, Tobia Zanon e Giovanni Zanovello. 


\title{
PARTE PRIMA
}

\section{Prassi esecutiva e riflessione teorica: su alcuni indizi musicali in Petrarca}

\author{
(Chiara Cappuccio)
}

\section{I.I. Lo stato della critica}

«Dove passa (nella prassi poetica medievale) il confine tra canto e non canto?» Quasi vent'anni fa Paul Zumthor si interrogava sulle caratteristiche di ciò che, all'interno di uno studio più generale sui rapporti tra la lettera e la voce nella letteratura medievale, veniva da lui definito come testo vocalizzato. ${ }^{2}$ A tutt'oggi, la domanda del filologo che pose al centro degli studi di letteratura medievale i fenomeni di oralità legati all' «ingerenza del corporeo nel grammaticale» continua a costituire un interrogativo imprescindibile all'interno delle ricerche sulla funzione della musica - intesa sia come riflessione teorica che come presenza reale - nei testi lirici romanzi appartenenti ai repertori profani medievali. Domanda che sia negli studi filologici che in quelli musicologici appare ormai indissolubilmente legata a quello che, dai saggi classici sull'argomento di De Bartholomaeis, Contini e Roncaglia, è diventato un topos della nostra tradizione critica, ossia il famigerato, e presunto, divorzio tra poesia e musica nella poesia d'arte italiana. ${ }^{3}$ Negli ultimi anni tale argomento ha visto un rinato interesse legato sia alle recenti scoperte relative ai frammenti che costituirebbero le «tracce di una tradizione sommersa» italiana conservata con musica che alla questione sempre aperta sul ruolo svolto dai siciliani nel processo di separazione della creazione poetica da quella musicale. ${ }^{4}$

2. Paul Zumthor, La lettera e la voce. Sulla «letteratura medievale» (1987), Bologna: Il Mulino, 1990 , p. 248.

3. Vincenzo De Bartholomaeis, Primordi della lirica d'arte in Italia, Torino: SEI, 1945, p. 121; Gianfranco ConTINI, Poeti del duecento, Milano-Napoli: Ricciardi, 1960, I, p. 45 e Id. Varianti e altra linguistica, Torino: Einaudi, 1970, p. 176. Aurelio RonCAGLIA, «Sul divorzio tra musica e poesia nel duecento italiano", in L'Ars Nova in Italia nel trecento, IV, Certaldo: Centro Studi sull'Ars nova italiana del trecento, p. 365-397.

4. Ottavio TibY, "La musica alla corte dell'imperatore Federico II», in Atti del Congresso Internazionale della poesia e della lingua italiana (Palermo, giugno 1951), Palermo: Pezzino, 1951. Nino PIRrotTA, «I poeti della scuola siciliana e la musica» in Poesia e Musica, Firenze: La Nuova Italia, 1994, p. 13-51. Roberto ANTONELLI, «La scuola poetica alla corte di Federico II», in Federico II e le scienze, Palermo: Sellerio, 1994, p. 308-323. Joachim SCHULZE, Sizilianische Kontrafakturen, Tubingen: Niemeyer, 1989. Per un ultimo aggiornamento della questione si veda: Francesco CARAPEZZA, «Un "genere” cantato della scuola poetica siciliana?», Nuova Rivista di Letteratura Italiana, II (1999), 2, p. 321-354. Ricordiamo inoltre il celebre saggio che inaugurò la questione siciliana: Istvan FRANK, "Poésie romane et Minnesang autour de Frédéric II. Essai sur les dëbuts de l'école sicilienne», Bollettino del Centro di Studi Filologici e Linguistici Siciliani, III, p. 51-83. Sul frammento piacentino vedi: "Tracce di una tradizione sommersa. I primi testi lirici italiani tra poesia e musica. Seminario di studi», Cremona, 19-20, Febbraio 2004, gli atti sono in corso di stampa; sulla carta ravennate: Alfredo STUSSI, "Versi d'amore in volgare tra la fine del XII e l'inizio del XIII», Cultura Neolatina, 69, Fasc. I, 1999, p. 1-69. 
Sono presenti, nell'opera di Petrarca, accenni alle riflessioni sulla musica caratteristiche dei testi di filosofia o di versificazione del tempo o sono presenti degli indizi lessicali che possano provare la continuità di un uso monodico o semplicemente "vocalizzato" dei testi lirici (illuminando così, retrospettivamente, il percorso compiuto dalla relazione tra musica e parola in Italia)? Il corpus petrarchesco, sottoposto ad una ricerca sistematica dei luoghi musicali in esso contenuti, può essere interrogato sui problemi relativi alla relazione tra dizione e armonia o a quelli della formalizzazione ritmica dei testi medievali, cioè a quei problemi legati alla nostra incipitaria domanda zumthoriana?

La prospettiva critica che si propone riguarda sia la ricerca di possibili testimonianze sulle pratiche musicali familiari al poeta che le presenze di eventuali frammenti di un discorso teorico. Intorno a questi due punti, che riconducono il primo alla ricostruzione di una prassi, il secondo al recupero di una teoria, si articoleranno le riflessioni nate dalla schedatura analitica dei riferimenti musicali presenti nell'intera produzione dell'autore.

I luoghi classici, interni ed esterni al corpus petrarchesco, su cui la critica ha tradizionalmente esercitato le proprie capacità esegetiche relative alla ricostruzione di una coscienza musicale dell'autore, sono costituiti: ${ }^{5} 1$ ) dai i riferimenti epistolari alle sue frequentazioni musicali — amici compositori ed esecutori, giudizi sulle determinate qualità dei cantori, riferimenti alla vita musicale del tempo, lettere di raccomandazione per taluni musici-, 2) dal passo del testamento, riportato nel titolo, in cui Petrarca lascia il suo liuto migliore all'amico Tommaso Bambasio ${ }^{6} 3$ ) ed infine dalle cronache e dalle biografie trecentesche che lo vogliono sicuro conoscitore di musica nonché dotato di una voce dolcemente intonata. ${ }^{7}$

5. Gli studi che iniziarono al principio del novecento a ricostruire i rapporti di Petrarca e la musica sono: Carlo CulCASI, Petrarca e la musica, Firenze: Bemporad 1911; Arnaldo BonaVENTURA, «Petrarca e la musica», Annali della cattedra petrarchesca diretti da Guido Paliotti, Accademia Petrarca di Lettere, Arte e Scienza di Arezzo, Vol. III, 1932, p. 25-43.

6. Ė stato recentemente messo in discussione da Stefano Campagnolo al congresso «Petrarca in musica», Arezzo, 18-20 Marzo 2004, i cui atti sono in corso di stampa, lo statuto di musicista di Bambasio. Da sempre considerato dalla critica come tale, Campagnolo dimostra come in realtà l'amico di Petrarca sia sicuramente un maestro d'armi come riportato da Senili, IV, 3 in cui Petrarca descrive a Pietro da Bologna le feste di Venezia per la conquista di Creta in cui Bambasio è chiamato a dirigere una giostra, mentre le sue competenze musicali non risulterebbe da nessuna testimonianza. Nel codice Vat. 3196 sono inoltre presenti due brevi postille che testimoniano un invio di sonetti a Bambasio.

7. Riportiamo qui alcune tra le più famose delle testimonianze biografiche e cronachistiche: Boccaccio riporta che la sua voce era così armoniosa «ut sint qui hunc audiendo concedant verum a cantibus Sirenarum sociorum ducis Naricii naves fuisse submersa, dum se a dulcedine prolationis istius quodammodo comperiant fore captos. Et alii sunt qui firma teneant fide quod eaedem apes, quae Platoni Ambrosioque dormientibus parvulis melle labia delenirunt, huic tymo cyrrhensi saturae etiam parvulo delinissent».

Dice inoltre che si dilettava «in musicalibus vero prout in fidicinis et cantilenis».

Donato degli Albazani: «Et ego ipse Stupeum rogavi dicere aliquid inter egregias dominas e cuius ore loquendo tanta suavitas procedebat, qua in mensis seu in cena derelinque- 
Nonostante le presenze su elencate, però, Petrarca evita quasi sempre, e quasi con cura, di trattare l'argomento musicale. Reticenza sorprendente se si considera che vi è una parte dell'epistolario dedicata alla corrispondenza con Philippe de Vitry (uno dei più rilevanti esponenti dell'ars nova francese, teorico, compositore, poeta e diplomatico) e con Socrate-Ludvig van Kempen (altro musicista legato al nuovo linguaggio musicale).

Lasciando da parte la questione del petrarchismo musicale, fenomeno successivo e rispondente ad altri principi di composizione e uso del testo lirico, gli elementi su elencati avrebbero fatto prevedere la presenza, all'interno soprattutto della produzione latina, di riflessioni e idee sulla scientia musicalis o almeno sulle pratiche esecutive del tempo, considerando il ruolo significativo che l'arte dei suoni aveva svolto nella formazione intellettuale medievale in quanto disciplina quadriviale; ed è soprattutto l'assenza del vocabolario concettuale di memoria agostiniana e boeziana, di solito presente, invece, nei riferimenti alla musica dei suoi predecessori, che costituisce forse la prima novità del nondiscorso di Petrarca sulla musica. ${ }^{8}$

Le ricerche più recenti di storia e filologia musicale spostano, invece, il centro delle ricerche su due aspetti principali; il primo riguarda, evidentemente, lo studio del madrigale Non al suo amante più Diana piacque, unico testo dell'autore presente nel canzoniere di cui esiste anche una trasmissione musicale e che studi recenti hanno dimostrato essere inizialmente composto per un'occasione musicale prima di vedere la sua sistemazione all'interno dei $R V F^{9}{ }^{\grave{\mathrm{E}}}$ l'unico testo su cui si ha una testimonianza sicura che attesta l'esistenza di una veste musicale che Petrarca dovette sicuramente conoscere e con cui dovette fare i conti nel momento della composizione del testo poetico. La musica, opera di Jacopo da Bologna, è presente, anonima dal punto di vista poetico,

bat omnes attentos ad ipsam solam facundiam; aliquando iocose recitabat inter dominas rytma vulgaria, quae mirabiliter erant auditu et per ipsum composita».

Filippo Villani: «Petrarcha doctus insuper lyra mire cecint, unde labores studii modeste levabat [...]. Fuit vocis sonorae atque reduntantis, soavitas tantae atque dulcedinis, ut nescirent etiam doctissimi ab eius collucutione discedere».

8. È sintomatico del fenomeno appena descritto la senile a Guglielmo Maramaldo (Senili, XI, 5 ) in cui Petrarca rovescia il postulato della gerarchia tripartita musicale boeziana che fonda tutta la speculazione teorico-musicale del medioevo. Parlando dello speciale diletto che prova ascoltando la soavità della voce di un musico che chiama italico Orfeo scrive che la musica istrumentalis è superiore alla quella coelestis.

9. Pierluigi PeTrobelLI, «Un leggiadretto velo e altre cose petrarchesche», Rivista Italiana di Musicologia, X, 1975, p. 32-45. Nello studio di Petrobelli vengono inoltre analizzate le varianti testuali esistenti nelle differenti redazioni del madrigale rispetto alla versione definitiva riportata dal Vat. Lat. 3195 e che testimonierebbero i vari sforzi di adattazione al tono del Canzoniere di un testo composto in una prospettiva di uso mondana e musicale. Sull'argomento si veda almeo anche Laura PAOLINO, "Ancora qualche nota sui madrigali di Petrarca (RVF 52, 54, 106, 121)», Italianistica, XXX, 2, 2001, p. 307-324; Guido CAPOVILLA, «I madrigali» (LII, LIV, CVI, CXX), (1983), in "Si vario stile», Studi sul Canzoniere di Petrarca, Modena: Mucchi, 1998, p. 47-90. e, sulla questione più generale dei madrigali del canzoniere: Massimo ZENARI, "In margine ai fragmenta LIV e CXXI di Petrarca», in Carmina semper et citharae cordi. Etudes de philologie et de metrique offertes a A. Menichetti, Géneve: Slatkine, p. 241-251. 
nel cod. Squarcialupi. Il secondo punto riguarda il riferimento a Confortino (Pro Confortino) del Codice Casanatese 924 e del Vat. Lat. 3196 con i relativi tentativi di identificazione condotti sulla figura del musico in questione. Probabilmente amico del poeta (se si accetta l'interpretazione classica della postilla per cui il musico, cui Petrarca avrebbe inviato un gruppo di ballate, ha addirittura la possibilità di scegliere tra esse quella che preferisce, e che occuperà quindi la prima posizione, Amor ch'in cielo e 'n gentil core alberghi,), ${ }^{10}$ si propende oggi a credere che sia la stessa persona citata nel celebre sonetto di Nicolò de' Rossi Io vidi ombre e vuy al paragone in cui compaiono i nomi, probabilmente più famosi, dei musici dell'epoca legati alla produzione lirica. ${ }^{11}$ Le ricerche su Confortino sono collegate alla questione più generale riguardante le identificazioni possibili dei nomi di musicisti presenti nei testi letterari medievali.

Ovviamente ricchissima, ma poco interessante ai fini della nostra ricerca, è la letteratura critica riguardante il fenomeno del madrigalismo musicale sviluppatosi sui testi del canzoniere; musicati da Villaert, Marenzio, de Rore, Caccini, Monteverdi, Palestrina ecc., i testi di Petrarca verranno considerati come garanzia dell'equilibrio poetico-musicale del nuovo genere polifonico. ${ }^{12}$

Sia la critica musicale tradizionale che quella più recente fondano spesso le loro analisi partendo dalla considerazione che l'autore vive ed opera in un momento di cruciale e definitiva trasformazione del linguaggio musicale: sono gli anni dell'elaborazione della teoria e della prassi compositiva polifonica e arsnovistica, e, a giudicare dagli amici che ha (Philippe de Vitry ne era uno dei più illustri teorici) non può non esserne al corrente (considerando, inoltre, che lo era già Dante). Inoltre, si evidenzia spesso il fatto che ci si muove negli anni dell'incipiente sperimentazione lirico-musicale delle rime per musica polifonica che, rendendo la parola definitivamente ancillare all'elaborazione melodica, romperà l'equilibrio monodico dell'intonazione dei testi poetici.

10. «hec in ordine retrogado adliteram, nisi fallor, ut hic dicyavi anno isto pro Confortino et unum aliud postea quod non curavi perficere ex his autem elegit, ipse ultimum quod hic est primum scripsi hoc ne elaberetur in totum que magna...»

Petrobelli riporta le due traduzioni possibili della postilla, considerando elegit o come parola completa o come con la $t$ come principio di un tandem o tamen per cui la prima è: "questi, in ordine retrogado, letteralmente qui sono, salvo errore, dettai questo anno per Confortino; e un altro poscia che non curai di finire. Tra questi egli scelse l'ultimo. Quello che qui è il primo lo scrissi perché non si perdesse interamente...", oppure: «Tra questi alla fine (o nondimeno) io stesso scelsi l'ultimo che qui è il primo. Lo scrissi perché non si perdesse interamente....», Pierluigi PeTrOBELli, op. cit., p. 42. La stessa ballata si trova ripetuta nel cod. Vat. Lat. 3196 con l'annotazione che segue: «1350. decembris. 26. inter meridiem et nonam. Sabato. per Confortinum».

11. Sul sonetto di Nicolo de'Rossi si veda almeno: Mahmoud SALEM ElSHEIKH, «I musicisti di Dante (Casella, Lippo, Scocchetto) in Nicolò de'Rossi», Studi danteschi, n. 48, 1970. p. 153-166.

12. L'ispirazione che Petrarca ha spesso fornito ai musicisti non si esaurisce unicamente nell'ambito madrigalistico. Nel XVIII sec. Haydn musica "Solo e pensoso», e, nel secolo successivo Schubert introdurra tre testi petrarcheschi tra i suoi Lieder. In seguito il poeta sarà ancora fonte di ispirazione compositiva per Liszt e Schönberg. 
Ma la trasformazione contrappuntistica del linguaggio e della composizione musicale, all'epoca di Petrarca, come sottolineato più volte da Petrobelli, è fenomeno marginale, elitario e circoscrivibile all'interno di ben delimitati centri di produzione e consumo, mentre la pratica ancora corrente a quest'altezza cronologica, a giudicare dalle testimonianze degli storici e dalle, anche se limitate, testimonianze letterarie che ci restano, doveva essere ancora quella legata alle modalità esecutive del testo, costituite dalla recitazione cantilenata e dall'intonazione monodica di matrice trobadorica (con il non poco rilevante passaggio della funzione musicale dal parametro compositivo a quello esecutivo).

Considerando quindi i risultati dei recenti studi su Petrarca e la musica e partendo dalla ricostruzione della vita musicale trecentesca italiana di Petrobelli, ripercorriamo le possibili testimonianze petrarchesche su una ancora viva e operante prassi di esecuzione del testo lirico.

\section{I.II. Sulla prassi: metrica e musica}

Sono presenti, soprattutto all'interno dell'epistolario, degli accenni alle abitudini lirico-musicali trecentesche? Petrarca si riferisce all'uso recitativo dei testi da lui composti, parla in due occasioni di giullari, esecutori e questuanti di ogni tipo che lo assillano con la richiesta di versi da musicare e, a volte, invia testi ad amici musicisti. Sembra quindi probabile che una forma di vocalizzazione del testo lirico, di carattere probabilmente cantilenato su delle corde di recita di tipo semiformulaico, fosse ancora in uso all'epoca di Petrarca ed è ad essa che egli si riferisce quando parla dell'esecuzione recitata dei suoi testi poetici. Che poi un musico potesse imbastirne un eventuale rivestimento musicale dipende dal fatto che, in tutta la poesia romanza precedente come ancora in Petrarca, la struttura metrica di un testo lirico continua a garantirne le possibilità esecutive anche dal punto di vista musicale. Ė la metrica che conserva all'interno delle diverse e nuove strutture la memoria e l'uso delle possibilità e virtualità musicali che un compositore professionista (un magister Casella per Dante o un Confortino per Petrarca) può volgere facilmente e rapidamente in musica forse proprio grazie a quelle formule di intonazione melodica adattabili ai differenti registri metrici che sopravvivevano nel Medioevo —e di cui sono probabile e posteriore testimonianza gli aeri, «stereotipi melodici che rimandano nel loro stesso titolo alla forma poetica corrispondente, e prendono il nome di aeri con accanto la specificazione del genere poetico cui si accompagnavano: aere da cantar sonetti, aere da cantar strambotti, ecc.» ${ }^{13}$

13. Pierluigi Petrobelli, «Poesia e musica», in Letteratura italiana. VI. Teatro, musica, tradizione dei classici, Torino: Einaudi 1986, p. 227-244. Il musicologo Francesco Rossi, nel suo intervento al congresso aretino su "Petrarca in musica» dal titolo "Vergine Bella e Dufay: dalla tradizione improvvisativa alla res facta», dimostra l'utilizzo da parte di Dufay, per la realizzazione della melodia con cui musicò il testo petrarchesco, di un aere, cioè di un nucleo melodico legato alla prassi improvvisativa delle intonazioni alla viola. La famosa composizione del polifonista costituirebbe così un ponte tra una pratica intonativa-improvvisativa 
Non solo il poeta non è più anche il compositore del testo musicale, come lo erano invece i trovatori, ma quest'ultimo non è neanche più considerabile come tale, cioè come un testo strutturato in vista di un preciso rapporto con quello poetico; la struttura metrica rimane ora il richiamo ultimo ad una sempre possibile musicabilità della parte letteraria.

Si può quindi ipotizzare la persistenza di un primo e usuale livello recitativo/cantilenato del testo - con tutte le considerazioni che il confine tra recitazione cantilenata e musica comporta quando si parla di pratiche di esecuzione e fruizione medievali (basti pensare ai codici virgiliani e ciceroniani neumati o alle testimonianze relative alle esecuzioni "cantate» di un testo come la Commedia di Dante) ${ }^{14}$ _ e un altro, possibile, che non incide più sulla composizione del testo, se non per quanto riguarda le scelte metriche, ma che si produce a partire e in forma indipendente da esso, relativo all'uso melodico e monodico che probabilmente perdurava ancora. ${ }^{15}$ Il fenomeno della composizione delle rime per musica e dei generi letterari musicali polifonici costituisce invece ancora un altro livello, in quanto fenomeno esclusivamente musicale in cui si organizza un preciso testo polifonico a più voci che si serve e si appoggia su un testo letterario la cui comprensione, comunicazione e trasmissione non è più però ad esso legata.

Che le forme di vocalizzazione dei testi lirici fossero al centro delle consuetudini esecutive sicuramente ancora all'inizio del XIV sec. viene confermato dal celebre passo del De vulgari eloquentia, in cui il momento creativo (actio), viene distinto da quello esecutivo (passio). ${ }^{16}$

legata alla tradizione musicale non scritta e l'organizzazione polifonica del pensiero musicale che acquisisce quasi da subito lo statuto della scrittura.

14. John AHERN, "Singing the book: Orality in the Reception of Dante's Comedy», Annals of Scholaship. Metastudies of the Humanities and Sacral Sciences, n. 2, 1981, p. 17-40. La presenza di strutture neumatiche che accompagnavano i testi virgiliani e ciceroniani nei codici medievali va messa in relazione con un luogo delle Senili, in cui Petrarca ricorda la precoce lettura «melodica» di Virgilio e Cicerone: "Ed illa quidem aetate nihil intelligere poteram, et sonoritas detenebat, tu quicquid aliud vel legerem vel audirem, raucum mihi, longeque dissonum videretur» (Sen., XV.1).

15. Sull'impossibilità di tracciare un confine tra le pratiche di recitazione, canto e cantilena sono fondamentali le pagine di Zumthor sull'essenza del testo vocalizzato. Qui l'autore, partendo dallo studio del cursus e della sequenza, individua i modelli classici della prassi di recitazione medievale in cui il canto si riferisce a omnes sonorum gradus per cui attori e oratori dovevano conoscere quest'arte. Riporta inoltre sia il prologo dell'Alexis sia il trecentesco Durmand le galois in cui i termini leggere, raccontare e cantare si dispongono in dittologie sinonimiche. Scrive l'autore: «Figure cumulative che evocano, congiungendo i due registri di parola, una totalità vocale. Importa poco che il limiti oscilli, nel corso del tempo, fra quello che l'orecchio percepisce come parola detta e quello che percepisce come canto(è in questa porspettiva che a Firenze nel ' 300 si cantavano le terzine della Commedia)». Sempre nello stesso testo Zumthor ricorda inoltre l'Art de dictier di Eustache Deschampe che alla fine del trecento distingue ancora tra la musica artificiale degli strumenti e quella naturale del parlato poetico.

16. Si veda sull'argomento l'importante contributo di Maria Sofia LANNUTTI, "Ars e scientia, actio e passio. Per l'interpretazione di alcuni passi del De vulgari elquentia», Studi Medievali, 2000, p. 1-38. 
Est enim cantio, secundum verum nominis significatum, ipse canendi actus vel passio, sicut lectio passio vel actus legendi. Sed divaricemus quod dictum est, utrum videlicet hec sit cantio prout est actus, vel prout est passio. Et circa hoc considerandum est quod cantio dupliciter accipi potest: uno modo secundum quod fabricatur ab autore suo, et sic est actio - et secundum istum modum Virgilius primo Eneidorum dicit Arma virumque cano-; alio modo secundum quod fabricata profertur vel ab autore vel ab alio quicunque sit, sive cum soni modulatione proferatur, sive non: et sic est passio. Nam tunc agitur; modo vero agere videtur in alium, et sic tunc alicuius actio, modo quoque passio alicuius videtur. ${ }^{17}$

De vulgari eloquentia, II, VIII, 3.

Le artes dictaminis cronologicamente più vicine al Petrarca, non ponendosi mai il problema di differenziare i testi musicabili da quelli non-musicabili, sembrano abbastanza esplicite sull'argomento; tutti i testi poetici erano suscettibili di un rivestimento musicale, sempre quando le scelte metrico-ritmiche del verso ne garantissero la possibilità. Sia il De vulgari eloquentia, che i trattati di versificazione che, come la Summa di Antonio da Tempo o l'anonimo Capitolum de vocibus applicatis verbis, tendono a fornire una descrizione pratica delle forme in vista di un uso, rappresentano una testimonianza eloquente delle profonde relazioni tra metrica e melodia. Nella trattazione della canzone, Dante lascia intendere più di una volta che la presenza della musica è una possibilità sempre garantita dalla adeguata organizzazione metrica di un testo. La musica diviene un parametro compositivo assorbito dalla struttura metrica. "Dicimus ergo quod omnis stantia ad quandam odam recipiendam armonizata est». L'ars musica, unica scientia ad assumere nella tradizione quadriviale i connotati di una forma d'arte, entra nella teoria del verso e, una volta assorbita nell'equilibrio ritmico e metrico della stanza, guida l'organizzazione del testo lirico. La forma strofica viene così descritta da Dante come determinata da precise proporzioni musicali, dal momento che la composizione di un testo prevede l'unione di rethorica, eloquentia e musica, cioè delle arti del trivio (che si occupano delle forme del discorso) e quelle del quadrivio (che invece hanno come oggetto la cognitio rerum).

L'ars di cui si occupa la trattatistica è, ovviamente, sempre quella vocale, erede dello stretto legame tra musica (ars e scientia al tempo) e poesia. Il musico, prima di divenire lo specialista trecentesco dell'arte dei suoni, è colui che esercita una capacità di giudizio razionale sull'unione tra musica e parole, la cui

17. «E a questo proposito bisogna tener presente che il termine canzone si può assumere in due sensi: in uno, in quanto è costruita dal suo creatore, e in tale senso è azione - è in questa accezione che Virgilio nel primo dell'Eneide dice Canto le armi e l'eroe-; in un altro in quanto, una volta costruita, venga recitata dal suo creatore o da chiunque altro, con o senza modulazione della melodia; e, in questo senso è passività. Perché in quel caso è agita, in quanto agisce invece su un altro, e così là si rivela come azione fatta, qui come azione subita da qualcuno». Dante Alighieri, Opere Minori, v. III, I, De vulgari eloquentia, a cura di Pier Vincenzo Mengaldo, Milano-Napoli: Ricciardi, 1979, p. 201. 
relazione è regolata del concetti di ritmo e di numerus (basti considerare che il De musica di Agostino è un trattato essenzialmente di metrica). ${ }^{18}$

Che la struttura metrica di un testo lirico costituisca il vincolo tra poesia e musica, lo scheletro sul quale si articolano l'andamento ritmico e la forma melodica di un testo lirico medievale, è stato più volte dimostrato dai recenti studi del musicologo e filologo Antoni Rossell che, raccogliendo l'eredità teorica di Ugo Sesini e Jorn Gruber, dimostra come nella redazione dei testi poetici medievali siano leggibili precise strategie metrico-melodiche di organizzazione del testo che spingono a considerare il metro come la sede di quest'organizzazione. ${ }^{19}$ Nei suoi studi sui sistemi di imitazione poetico-musicale tra testi romanzi, Rossell ricorda spesso come le norme per una corretta contraffazione metrico-melodica dei testi lirici riassunte nel trattatello anonimo posto al principio del canzoniere Colucci-Brancuti (Art de trobar) dimostrano come la struttura metrica e quella melodica siano considerate un' unica variabile compositiva. $^{20}$

Gli strettissimi legami tra struttura metrica e organizzazione melodica sembrano talmente radicati nella composizione lirica da rendere difficile una loro netta separazione in sede teorica. Per la trattatistica classica erano indissolubilmente legate l'una a l'altra e ciò accadeva anche nella prassi musicale più generale, se si pensa che, per es., i modi ritmici gregoriani altro non erano che la veste musicale del sistema metrico greco. J. Gruber, come riporta sempre Rossell, si spinge addirittura oltre, dimostrando come la lirica cortese medievale non possedesse un termine per indicare la struttura metrica. Considerando il testo di No sap chantar qui so non di, la canso de lonh del trovatore Jaufré Rudel, e analizzandolo sia dal punto di vista lessicale che da quello intertestuale, Gruber giunge alla conclusione - accettata dalla critica filologica - che la parola occitana so indica allo stesso tempo la melodia e la struttura metrica. ${ }^{21}$

Mentre però nella composizione di matrice trobadorica l'elaborazione metrico-melodica costituisce un parametro compositivo (la opción poetica, di cui parla spesso Rossell) in cui sperimentazione metrica e musicale dipendono l'una dall'altra, nella lirica italiana sembra già maturo il passaggio verso il ridimensionamento della funzione musicale in formule di intonazione classificabili in base alla scelta del metro. Tutta la pratica relativa alle contraffazioni metrico-musicali tra testi romanzi rende esplicita questa ipotesi.

18. Per Boezio, come nota sempre M.S. Lannutti, il musico è addirittura superiore al poeta, cui manca un grado sufficiente di consapevolezza teorica, dal momento che su di lui agisce maggiormente il fattore istinctum.

19. Antoni Rossell, in La lirica galego-portoghese, saggi di metrica e musica comparata, Roma: Carocci, 2003.

20. Nel breve trattato di poetica contenuto nel Cancioneiro Colocci-Brancuti si definisce l'arte de seguir distinguendo tra tre diversi livelli di imitazione; il primo, in cui vengono adattati al nuovo componimento solo la melodia e la struttura sillabica del precedente componimento, il secondo, che prevede un'imitazione di versi, rime e struttura strofica del modello scelto, ed il terzo che considera il livello lessicale come portatore di più significati.

21. Jorn Gruber, Die Dialektik des Trobar, Max Niemeyer Verlag, 1983. 
Ancora all'altezza della stesura del De vulgari eloquentia Dante, consapevole della novità teorica costituita dalla polifonia, e al tempo stesso erede sia della tradizione boeziano-quadriviale sia di quella formulare-improvvisativa di ascendenza trobadorica, rende chiaro il carattere di vincolo formale tra poesia e musica incarnato dall'organizzazione metrica di un testo. Per Dante infatti ogni testo, se composto secondo le scelte e i parametri che elencherà all'interno del trattato, è sempre musicabile da un professionista della disciplina. ${ }^{22}$

Petrarca, nonostante le sue reticenze sulla musica, può rappresentare una situazione esemplare dei diversi comportamenti poetico-musicali che la lirica in volgare incarna nel ' 300 , testimoniando allo stesso tempo il perdurare di pratiche di esecuzione vocale e di rivestimento monodico dei testi volgari e, parallelamente, il compimento di quella tendenza a svincolarsi dall'associazione con la musica che la poesia volgare italiana intraprende tra la fine del XIII e l'inizio del XIV. Su un altro versante sia pratico che teorico, come già specificato, vanno collocate le incipienti sperimentazioni polifoniche su testi volgari che produrranno un fenomeno completamente diverso in cui tra il testo poetico e l'organizzazione melodica delle voci non c'è praticamente più nessun legame e che è fenomeno ora squisitamente musicale.

\section{I.III. Sulla teoria: da Boezio a Orfeo}

La mancanza di considerazioni teoriche che caratterizza l'opera latina di Petrar$\mathrm{ca}$, - silenzio tanto più eclatante se paragonato alla varietà del discorso musicale dantesco- può considerarsi più eloquente di quanto sembri. Si possono

22. Sono molte le posizioni critiche che si potrebbero riportare a conferma dell'unita di metrica e musica nella composizione lirica medievale. Nel trattare l'argomento in questione ci sembra imprescindibile ricordare, almeno in nota, la figura del grande musicologo e filologo romanzo Ugo Sesini, che fu tra i primi a parlare della libera monodia e della variabilità dell'esecuzione musicale che caratterizzavano i testi lirici romanzi (prima di lui, infatti, ai testi occitani e francesi venivano applicati i criteri di lettura derivati dai modi grogoriani). Per Sesini, metrica poetica e leggi ritmiche musicali sono regolate dagli stessi elementi: il concetto di tempo primo, il senso arsico e tetico che caratterizza ritmicamente il procedere sia del verso che della melodia, il raggruppamento dei tempi primi in ritmi binari e ternari. Studiando il rapporto tra ritmo verbale e ritmo musicale all'interno della metrica classica e romanza, l'autore ricerca, nel momento storico che segna il lungo e articolato passaggio dalla prima alla seconda, la genesi ritmica del verso tonico ed il formarsi del rapporto tra verso poetico e ritmo musicale che sostanzierà la lirica medievale romanza. Nel passaggio dal verso quantitativo latino a quello tonico romanzo, la graduale scomparsa del senso di quantità, accompagnata da una lenta trasformazione dell'accento da melodico ad intensivo, produce un uniformità in quanto alla durata metrica di tutte le sillabe. L'esistenza di «un tempo primo musicale equivalente alla sillaba metrica, alla quale tutte le figure neumatiche, dal punctum al gruppo complesso, dovevano sottostare», nonché «la corrispondenza tra verso poetico e frase melodica, per cui ad un membro poetico corrisponde una distinctio musicale delimitante la quantità ritmica complessiva della frase» conducono Sesini alla constatazione che la ritmica verbale-poetica e quella ritmicomusicale obbediscono agli stessi principi generali (Ugo SESINI, Le melodie trobadoriche nel canzoniere provenzale della Biblioteca Ambrosina $R 71$ sup., Torino: Giovanni Chiantore, 1942, p. 57). 
isolare due tendenze principali che guidano abitualmente le considerazioni in materia musicale di Petrarca: la prima riguarda un topos della letteratura musicale (e medica) fin dall'antichità, e cioè l'interesse per gli effetti che la musica produce sugli ascoltatori, la seconda riguarda il riconoscimento della musica come valore autonomo, nella sua speculazione teorica, e della professionalità del musico come interprete di un linguaggio specialistico.

Variamente orchestrati intorno al tema orfico, i riferimenti sui prodigiosi e potenti effetti della prima fa le arti sono presenti quasi ogni qualvolta Petrarca parli di musica. Tali effetti non si producono solo sull'animo umano (come spesso accade ai personaggi della Commedia); quando la musica è dolce (l'aggettivazione è topica) essa ha un effetto su tutto il creato, piante, animali ecc. La ripresa del motivo mitico costituisce un aspetto rilevante del paesaggio musicale petrarchesco, un aspetto nuovo rispetto a Dante dove la musica, almeno quella relativa ai primi due aspetti della classificazione boeziana - instrumentalis e la humana - sembra strettamente ancorata alla sfera dei sensi. In Petrarca la musica agisce sui sensi dell'ascoltatore ma anche sull'intero universo.

Inoltre, sono quasi del tutto assenti gli abituali riferimenti alla teoria boeziana, e soprattutto risalta la rimozione della famosa tripartizione della musica in instrumentalis, humana e coelestis, che informa gran parte della teoria filosofico-musicale medievale e con tale assenza (fatta eccezione per i pochi accenni alla musica delle sfere, ed alla diversità di posizioni che la filosofia tomistica scatena sull'argomento) si celebra il distacco, che sarà tipico della nuova trattatistica polifonica — nonché di tutto l'umanesimo- dalla trattazione musicale d'impostazione classica e medievale, in cui la musica è fondamentalmente numerus.

Petrarca sembra preoccuparsi soprattutto di rivisitare il motivo legato al tema orfico degli effetti che la musica esercita su tutto il creato. Fino a Dante invece gli effetti della musica erano analizzati in una prospettiva sempre psicologica, diversificando mediante l'aggettivazione le conseguenze positive o negative che essa poteva produrre. I sentimenti di eccitazione o di calma che la musica aveva la capacità di infondere rientravano nella teoria pitagorica che aveva classificato gli effetti prodotti dall'ascolto dei diversi modi della scala musicale greca (idea, tra l'altro, mai definitivamente scomparsa e ancora presente nella teoria zarliniana dell'ethos modale o anche in quella tonale romantica). Esistevano quindi diversi tipi di musica a seconda degli effetti che si volevano raggiungere. Dante, per es., quando intende parlare di una musica psicologicamente e socialmente positiva la connota sempre con un'aggettivazione derivata dalla sfera concettuale legata alla dolcezza e la soavità; dolci sono la voce, il timbro, la qualità del canto e delle armonie.

In Petrarca, invece, questa dicotomia sembra risolversi in una più generale contrapposizione tra musica sacra e profana, per cui la pratica musicale profana è connotata come frivola e vana, esercitando un forte potere sui sensi, e quella sacra come alta e degna. Il restringimento delle riflessioni teoriche sulla musica dell'autore all'antitesi sacro-profano sembra attestato: 1) dal cap. XXIII 
del De Remedis, De cantu et dulcedine musicae, in cui la Ragione, dopo aver distinto tra i vari effetti che la musica può suscitare nell'animo umano, rende omaggio alla musica, ma solo a quella che muove alla vita "divota e santa». 2) Dalla I egloga, in cui Silvio e Monico incarnano la dialettica tra musica sacra e profana. 3) Dal De Ocio (II), in cui si ripropone la frattura tra la vita mondana «delectatur jocis et cantibus» e quella monastica in cui i monaci lodano Dio «moestis suspiriis et semper duris accentibus». 4) Da alcune familiari, in cui la musica profana diventa simbolo della sensualità e del femminile-sirenico e si contrappone a quella sacra dei salmi e delle litanie (Fam. XXII.10, X.3 e Sen. III.8), e viene sancito infine dal testamento, in cui l'autore, dopo aver lasciato il suo leutum bonum all'amico Tommaso Bambasio prega quest'ultimo di usarlo per intonare musiche sacre e non profane.

Rispetto alla profonda articolazione concettuale cui la dialettica tra musica sacra e profana da vita nell'opera dantesca e segnatamente nell'architettura della Commedia (icasticamente rappresentata dal II canto del Purgatorio, di cui parleremo in seguito) la prospettiva petrarchesca sembra ristretta ad una generica contrapposizione tra una positività di finalità e intenti musicali riassunta dalla produzione gregoriana e ambrosiana ed una frivolezza e vacuità di contenuti morali espressa dalla produzione musicale laica legata ad occasioni mondane di produzione e consumo. L'unica deroga al paradigma dialettico sacro-profano è rappresentata dalla visione mitica della musica, tutta concentrata sugli effetti quasi panteistici da essa prodotti.

Sembra quindi che Petrarca sviluppi il suo discorso sulla musica in una prospettiva fortemente periferica rispetto ad altre urgenze filosofiche e morali e che essa si limiti a spostare e riproporre alcune di esse in un altra sfera concettuale ed espressiva. Il confronto tra una prospettiva di uso musicale laico e mondano ed una di tipo sacro sembra riflettere una dialettica morale e psicologica che permea l'intera produzione petrarchesca e che trova altrove le sue motivazioni etiche più profonde e i suoi sviluppi artistici meglio riusciti. Solo il tempo del mito può costituire una felice deroga al paradigma in questione senza comportare l'emergere di altre contraddizioni teoriche.

Il restringimento della prospettiva teorico-musicale relativa alla dialettica sacro-profano rispecchia in realtà un atteggiamento culturale più generale di Petrarca nei confronti della disciplina musicale. Il paragone, quasi obbligato, con Dante rende immediatamente visibile la trasformazione in atto. Sia all'interno della sua produzione teorica che nell'organizzazione della Commedia, la musica viene trattata sulla scia delle diverse prospettive speculative e pratiche cui l'ars musica era pervenuta. Il discorso musicale dantesco è vario ed articolato e più che essere ingabbiato nelle maglie di una prospettiva teorica cui dovrebbe essere funzionale sembra seguire gli intricati sviluppi cui tale disciplina era giunta. La musica è sapere teorico nel Convivio e, soprattutto, nel De vulgari eloquentia (fortemente presente nell'intera trattazione del II libro), è teoria filosofica e poetica ma anche pratica di esecuzione (e composizione) lirica profana, cui i vari accenni ai trovatori sembrano a volte far riferimento. È teoria del verso e pratica di esecuzione. 
Nella Commedia trovano spazio monodia e polifonia, musica sacra e profana, accenni a tecniche di composizione ed una summa delle posizioni teorico-filosofiche dell'aristotelismo musicale. Ci sono trovatori, il magister Casella, un liutaio ma anche inni e salmi, nonché la forte presenza dell'eredità concettuale agostiniano-boeziana sulla musica e il confronto tra queste presenze non si risolve nel paradigma sacro-positivo $v$ s profano-frivolo e mondano ma ripercorre nelle sue contraddizioni gli esiti e gli sviluppi della teoria e della pratica musicale degli ultimi secoli. La musica è ancora essenzialmente numerus, sapere di derivazione pitagorico-quadriviale cui Dante sembra pienamente ancorato pur seguendone gli sviluppi teorici e pratici. Ricercando una pretesa completezza ed esaustività sull'argomento l'autore della Commedia appare aggiornato sulle moderne innovazioni teoriche e pratiche del linguaggio musicale. Boezio può quindi convivere con la polifonia, come la presenza di inni e salmi con quella di Casella o del mestre dels trobadors.

In Petrarca invece sopravvive ben poco che possa rievocare l'organizzazione scientifica quadriviale della teoria musicale; l'ars musica non è più la forma con cui i numeri agiscono sul tempo e non è neanche più legata, in una prospettiva speculativa, alla teoria del verso e all'organizzazione metrica del testo poetico, come invece lo era in Dante. Da un lato essa è già una disciplina teorica specialistica e completamente autonoma, dall'altra è pratica di fruizione del testo poetico. Il restringimento prospettico dell'interesse musicale petrarchesco sembra riflettere quel fenomeno più generale che porterà ad una profon$\mathrm{da}$ trasformazione del linguaggio musicale nel passaggio tra ars antiqua ed ars nova. Ed è in tale ottica che Petrarca lo considera come un sapere, ed un linguaggio altro, da lasciare ai suoi amici addetti ai lavori, o come pratica esecutiva, ancora intonata monodicamente o già, a volte, organizzata polifonicamente. E così che si può spiegare la prospettiva musicale che emerge dalla lettura dell'epistolario in cui è spesso presente il tema dell'esaltazione della bravura di alcuni musici e la celebrazione della disciplina come completamente professionalizzata e come valore autonomo, frutto di una perizia specialistica, di talento e virtuosismo.

Da un lato Petrarca percepisce probabilmente la musica Philippe de Vitry o di Socrate-van Kempen in una prospettiva già di separata, elegante, mondana e completa separazione rispetto al fare poetico e alla teoria filosofica, come sapere autonomo e specialistico dall'altro sembra ancora partecipare delle pratiche, sicuramente ancillari rispetto alla scrittura, di uso monodico del testo lirico.

\section{Conclusioni}

Nello svolgersi di queste riflessioni sono stati privilegiati due aspetti relativi al rapporto di Petrarca con la musica: il primo riguardante la presenza di possibili riferimenti che potessero testimoniare la sopravvivenza delle pratiche di intonazione monodica dei testi lirici ancora all'altezza cronologica in cui si colloca la produzione dell'autore ed il secondo volto a ripercorrere i luoghi 
della produzione petrarchesca che permettessero di abbozzare una possibile visione teorica dell'autore sull'argomento musicale.

Su quest'ultimo punto, partendo dal confronto con la posizione dantesca sulla musica, si è evidenziata una netta frattura nella linea teorico-filosofico di ascendenza agostiniano-boeziana che sostanziava nel Medioevo qualsiasi discorso sull'ars musica. Se per Dante il discorso sulla musica è analizzabile in base a questa doppia prospettiva - una, lirica, che rivisita il percorso teorico e pratico dell'unione che tra mot e so aveva sostanziato le origini della poesia romanza, e l'altra, teorico-filosofica che, rimettendosi alla teoria quadriviale della musica come scientia, aveva caratterizzato per secoli l'insegnamento della disciplina in questione - in Petrarca esso sembra decisamente più esterno, ornamentale e legato al mito. Egli coltiva probabilmente una qualche pratica musicale, è amico di importanti musicisti e teorici, riconosce il valore profondo della musica sull'animo umano ma considera questa disciplina come separata, e sicuramente inferiore (almeno a giudicare da quanto dice nel capitolo del De remedis dedicato al canto e alla dolcezza della musica), rispetto a quella letteraria.

Rispetto invece alle tracce di sopravvivenze di abitudini musicali monodiche, sebbene Petrarca non parli esplicitamente di esecuzioni musicali delle proprie nugae, si riferisce comunque spesso a delle pratiche di vocalizzazione del testo poetico che rimandano alle forme di esecuzione orale dei testi letterari su cui forse venivano talvolta applicate quelle formule melodiche variamente adattabili alle diverse strutture metriche che costituivano degli stereotipi musicali registrati da tardive documentazioni che vanno sotto il nome di aeri $d i$ cantar sonetti, strambotti ecc. e che probabilmente venivano applicati in un secondo momento e indipendentemente dall'autore.

Per il musicologo F.A. Gallo, l'episodio musicale che sostanzia il II canto del Purgatorio fornisce una testimonianza di quella che probabilmente costituiva la pratica musicale prevalente nel medioevo romanzo. L'esecuzione musicale della seconda canzone del Convivio ad opera del musico Casella, che costituisce il nucleo dell'incontro tra Dante e l'amico magister, confermerebbe l'ipotesi che la linea melodica che accompagnava il testo poetico altro non era, almeno a questa altezza cronologica, che una modalità di esecuzione del testo poetico. Non si tratterebbe perciò di un testo musicale che entra in un preciso rapporto con quello poetico ma di una possibilità esecutiva legata ormai unicamente al momento dell'esecuzione e fruizione del testo lirico. La fenomenologia di tale pratica è evidentemente complessa ed articolata: legata alla memoria e all' $u$ $s o$, le intonazioni monodiche risentono inevitabilmente sia della «variabilità che impronta le esecuzioni che della rarità del ricorso alla scrittura». È in questo contesto che Gallo colloca i riferimenti presenti nei testi poetici in volgare che costituiscono ormai dei punti di riferimento obbligati per qualsiasi analisi sulla relazione tra musica e poesia nei repertori profani del XIII e XIV sec. relativi all'Italia: le misteriose figure di Casella, Scocchetto e Minuccio d'Arezzo, ${ }^{23}$ 
«Troilo cantore» che intona Cino nel Filostrato, ${ }^{24}$ la testimonianza del perduto codice Boccoliniano secondo la quale la ballata dantesca Deh, Violetta, che in ombra d'amore, avrebbe accolto il suono di Scocchetto (che, secondo Pirrotta, forse era anche poeta dal momento che lo stesso codice gli attribuisce una ballata), il problematico rivestimento musicale che il doppio sonetto dantesco, Se Lippo amico, inviato forse all'amico Lippo Pasci de'Bardi, invoca per la stanza di canzone Lo mio servente core, ed infine la rubrica del Vat. Lat. 3214 (c. 149) che attribuisce ad un Casella la musica di Lontana dimoranza di Lemmo Orlandi. ${ }^{25}$ All'interno dei più generali sistemi di trasmissione orale dei testi letterari, la relazione tra composizione poetica e intonazione melodica sembra connotare un uso mondano dei testi poetici dai trovatori fino a Petrarca, in una prospettiva che conserva delle caratteristiche comuni, al di là dei profondi cambiamenti che certamente caratterizzano l'itinerario poetico romanzo. Dal petrarchismo musicale in poi, e cioè dal trionfo delle pratiche di scrittura arsnovistiche che fondano il madrigalismo italiano e fiammingo, questo secolare rapporto tra poesia e melodia subisce una profonda e sostanziale modificazione: il testo poetico diventa ora una delle possibili modalità esecutive del testo musicale e la poesia di Petrarca ne diventa il banco di prova (un segnale esplicito della rivoluzione del linguaggio lirico-musicale post-petrarchesco viene generalmente rintracciata nella trasmissione madrigalistica, anonima nel testo poetico e generalmente affidata unicamente a codici musicali). Come abbiamo già fatto notare, sulla scia delle considerazioni di Petrobelli, la rivoluzione del linguaggio musicale costituita dall'avvento pratico e teorico della polifonia misurata, rappresenta, ancora ai tempi della produzione petrarchesca, un fenomeno elitario, isolato e limitato e pochi centri di produzione e sperimentazione musicale. La monodia libera che caratterizzava le intonazioni melodiche dei testi poetici, continuava ad essere, con ogni probabilità, la pratica più diffusa di intonazione di tali testi, ancora ai tempi di Petrarca — stando alla trattatistica contemporanea- di contro ad un sapere musicale sicuramente più agguerrito e specialistico dal punto di vista normativo e teorico. Se, prima dell'avvento della polifonia, quello musicale era un sapere quadrivial-filosofico e quindi in grado di far convergere su di esso gli interessi di un pubblico non necessariamente di addetti ai lavori, ora comincia a trasformarsi in un sapere e un linguaggio sempre più specializzati. I molti silenzi di Petrarca sulla musica, proprio in quei punti in cui ci si aspetterebbe un approfondimento

24. Santorre DebenedetTI, «Troilo Cantore», Giornale storico della letteratura italiana, 66, 1915, p. 414-425.

25. Sulla questione della «vestizione» delle ballate si veda almeno la sintesi riportata da Claudio GIUNTA, Versi a un destinatario. Saggio sulla poesia italiana nel Medioevo, Bologna: Il Mulino, 2002, p. 158-163. L'autore, ripercorrendo i momenti principali della questione, propende decisamente per un'interpretazione musicale del fenomeno delle collaborazioni diacroniche testimoniato dall'invio di sonetti con richieste di rivestimento per ballate o stanze di canzoni (nel caso dantesco), di contro all'altra interpretazione, di matrice bembiana, secondo la quale tale rivestimento significherebbe la richiesta di aggiunte di altre stanze alla ballata monostrofica. 
almeno sulle questioni teoriche più dibattute, diventano eloquenti: da un lato si riconosce la specificità e il valore autonomo di un linguaggio teorico sempre più lontano dalle origini pitagorico-boeziane, dall'altro il perdurare di pratiche mondane e condivise di consumo dei testi lirici, viene forse proprio confermato da questi silenzi, dal momento che i rapidi accenni alle pratiche di vocalizzazione dei testi bastavano a lasciare intendere che le consuetudini della monodia lirica e della recitazione ritmica e cantilenata continuavano secondo gli usi del tempo. Ci sembra quindi che il paradigma critico che vorrebbe un Petrarca fondamentalmente disinteressato alle questioni musicali, e in generale ai linguaggi altri, e al contrario la pratica compositiva lirico madrigalistica come fortemente dipendente da Petrarca —ossia una chiusura di Petrarca verso la musica e, viceversa un «interesse» della musica per Petrarcapossa essere interpretato in una prospettiva in cui i silenzi dell'autore sull'argomento significano coscienza delle continuità e delle trasformazioni del linguaggio, della teoria e della pratica musicale trecentesca. 


\section{PARTE SECONDA}

\section{La musica e la poesia volgare di Petrarca}

(Luca Zuliani)

Per i rapporti fra poesia volgare e musica nel Medioevo è possibile individuare una sorta di opinione vulgata, quella che più spesso è riportata da manuali e trattazioni generali: la lirica italiana si distingue per un precocissimo divorzio fra poesia e musica, già delineato per i poeti della scuola siciliana, ai quali si deve

l'iniziativa, tanto vivace rispetto ai provenzali classici, di avere in tutto disgiunto la poesia dalla musica [...]. Con questo instaurano il divorzio così italiano (onde poi europeo) di alta poesia e musica che la collaborazione d'un qualche "magister Casella» («sonum dedit») a libretti più che mai autonomi sopraggiunge semmai perentoriamente a sancire. ${ }^{1}$

All'altezza di Petrarca questo processo appare ormai del tutto compiuto. Petrarca invero si dichiara spesso appassionato di musica, e fra i suoi amici più cari conta alcuni celebri musicisti colti, a cominciare da «Socrate», il fiammingo Ludwig van Kempen. Vi sono inoltre nel Canzoniere poesie forse scritte in funzione di una melodia: i madrigali e meno facilmente le ballate, che potevano essere musicate e intonate a più voci nell'ambito dell'ars nova, la musica polifonica fiorita in Francia e Italia nel corso del '300. Vi è poi testimonianza di alcune ballate extravaganti inviate a un musicista di nome Confortino, ma riguardo al Canzoniere si incontra spesso nella moderna critica l'asserzione che solo un testo fu musicato da un contemporaneo: il madrigale Non al suo amante, da parte di Jacopo da Bologna. È infatti l'unico che sia conservato (anonimo, come di consueto) in un codice musicale trecentesco. ${ }^{2}$ Le forme metriche più praticate nel Canzoniere, sonetti e canzoni, appaiono invece lontane da un possibile uso musicale.

Un esame ravvicinato degli scritti di Petrarca conferma questa tesi: appare infatti chiaro ch'egli associa la sua poesia alla recitazione e non al canto. Ciò avviene innanzitutto all'interno del Canzoniere, quando accenna all'atto del poetare abbandonando il tradizionale uso del verbo cantare come proprio dell'enunciazione dei versi. Per citare un esempio fra tanti:

Quel foco è morto, e 'l copre un picciol marmo:

che se col tempo fossi ito avanzando

(come già in altri) infino a la vecchiezza,

1. Gianfranco CONTINI, «Preliminari sulla lingua del Petrarca», in ID., Varianti e altra linguistica, Torino: Einaudi, 1970, p. 176.

2. Il madrigale è nel codice Squarcialupi della biblioteca Laurenziana e solo la contemporanea presenza nel Canzoniere permette di attribuirlo a Petrarca. 
di rime armato, ond'oggi mi disarmo, con stil canuto avrei fatto parlando romper le pietre, et pianger di dolcezza.

\section{RVF 304, 9-14}

Allo stesso modo, nelle prose latine, le pochissime volte che Petrarca si cura di trattare la poesia volgare, sua o altrui, menziona la recitazione come unica possibile fruizione: un esempio è la Senile V, 2 a Boccaccio, quando ammette di dare talvolta qualche verso in volgare ad un volgare et vulgatum genus di ben noti personaggi che vanno in giro per le corti «vestiti [...] carminibus alienis»" 3 e questi versi «ingenti espressione pronuntiant», ossia "van declamando con artifizio di molta espressione, e dai Signori ne hanno in ricambio favori, denari, vestimenta, ed altri regali de' così fatti». ${ }^{4}$

Anche gli stessi giullari parrebbero recitare: ad esempio "commode vulgaria recitantis", "abile declamatore di testi volgari», è definito il giullare che consegna a voce un sonetto a Petrarca nella rubrica che nel codice Vat. Barb. Lat. 56 precede la lettera dispersa 24. Sul suo modo di recitare Petrarca scherza nella risposta. ${ }^{5}$

Del resto Petrarca, sia pure nella consueta stilizzazione delle sue pene d'amore, pare accennare a una modalità di composizione dei suoi cantica volgari tramite la recitazione a bassa voce, quando scrive che, nella solitudine agreste,

erompendo dalla mia bocca, la fiamma del cuore riempiva il cielo e le valli di un mormorio [murmure] infelice, ma, come ad alcuni parve, anche dolce; da ciò nacquero quelle rime in volgare dei miei giovanili tormenti [illa vulgaria iuvenilium laborum meorum cantica] dei quali oggi mi vergogno e mi pento, ma che pur sono assai gradite, come possiamo vedere, a coloro che sono colpiti dallo stesso male. ${ }^{6}$

Di una sua pratica musicale di qualunque tipo Petrarca invece non parla mai. È indicativo un luogo spesso citato da chi cerca accenni di tal genere in Petrarca: nell'Ecloga III del Bucolicum carmen Stupeus, ossia Petrarca stesso, dice a Dafne, ossia Laura, che per conquistarla ad un certo punto si mise, con

3. Pétrarque, Lettres de la vieillesse IV-VII, Paris: Le Belles Lettres, 2003, p. 127; e così la successiva.

4. Traduzione di G. Fracassetti, in F.P., Lettere senili, (a cura di Giuseppe Fracassetti), Firenze: Le Monnier, 1869-70. Il verbo pronuntiare suggerisce, ma non richiede espressamente che si tratti di recitazione e non canto: è l'unione col sintagma ingenti espressione che fa propendere decisamente per tale ipotesi.

5. Affidando la risposta in prosa latina al giullare, che aveva voce "quodammodo asperam et acutam», Petrarca lo ammonisce di riferirla "viva voce, sed non aspera ut solitus es: suaviter, oro te, sine clamore cerebrifago et sine accentibus horrificis; denique non barbarice, queso, sed italice» (F. PETRARCA, Lettere disperse, varie e miscellanee, a cura di Alessandro Pancheri, Parma: Fondazione Pietro Bembo / Ugo Guanda Editore, 1994. p. 166-169).

6. Ep. Fam. VIII, 3, 13, tradotta in F. Petrarca, Epistole, a cura di Ugo Dotti, Torino: UTET, 1968, p. 203. 
successo, a studiare musica. ${ }^{7}$ Ma poco più sotto afferma di avere proseguito in ciò poiché aveva ricevuto anche la solenne approvazione di Argus, ${ }^{8}$ ossia, come nell'ecloga precedente, del re Roberto di Napoli: ciò appare quindi come un'allusione alla sua laurea poetica e tale attività «musicale» si rivela un'allusione alla sua poesia, e in particolare, come di consueto, alla sua poesia latina.

Secondo una fonte ottocentesca, appunto in un commento al Bucolicum Carmen Donato degli Albanzani, l'amico di Petrarca a cui è dedicato il De sui ipsius et aliorum ignorantia, riferisce di avere spesso ascoltato Petrarca recitare i suoi componimenti volgari:

...et ego ipse Stupeum [ossia Petrarca, come si è già accennato] rogavi dicere aliquid inter egregias dominas existentem, e cuius ore loquendo tanta suavitas procedebat, quod in mensis seu in cena cibus derelinquebatur omnes attenti ad ipsam solam facundiam, aliquando iocose recitabat inter dominas rithma vulgaria que mirabilia erant auditu et per ipsum composita.?

Quanto abbiamo brevemente esposto, come si diceva, corrisponde all'opinione vulgata: nella lirica italiana fin dai primordi vi sarebbe stato un «divorzio" fra poesia e musica. Ma ora passeremo in rassegna alcuni elementi difficili da far entrare in questo quadro. Innanzitutto riguardo all' Ars nova come principale destinazione dei testi per musica: come scrive Petrobelli,

le forme polifoniche su testi in volgare (tutto il repertorio dell'Ars Nova italiana, con le sue appendici che si prolungano sin quasi alla prima metà del ‘400) ebbero un uso ed una diffusione in aree geografiche e culturali estremamente limitate; furono, in una parola, appannaggio ed espressione di alcune corti (Scaligeri e Carraresi, Malatesta) e di alcune città (Verona, Padova, Firenze). ${ }^{10}$

Quindi è un errore di prospettiva supporre che questa pratica musicale d'élite, affermatasi solo nel '300 e coltivata in ambienti culturalmente e geograficamente ristretti, fosse al tempo di Petrarca il modo usuale di associare versi e musica. Come spesso ricordano i musicologi, vi era invece una pratica capillarmente diffusa di intonazione musicale monodica con l'accompagnamento del liuto o della viola. A differenza dell'Ars nova, che talvolta, ma non sempre, giungeva ad essere fermata sulla carta, questa musica in Italia era tramandata

7. «Haud tacuisse velim, quod, cum mea pauca putarem | Posse placere tibi, studui si musica forte | Ars michi ferret opem, quod te sonus atque camene, | Non auri fulgor caperet», v. 5962 dell'Egl. III in F.P., Bucolicum Carmen, translated and annotated by Thomas Goddard BERGIN, New Haven and London: Yale University Press, 1974.

8. Vv. 69-70: «Nec nata prius fiducia nostri est, | Quam sacer ille michi: —Cane fidens, — diceret Argus».

9. Da Attilio HorTIS, Scritti inediti di Francesco Petrarca, Trieste: Tipografia del Lloyd Austroungarico, 1874, p. 232. Il ruolo di Donato nei commenti alle egloghe è comunque tuttora non chiaro, né il volume qui citato riporta molto più del presente brano.

10. Da Pierluigi Petrobelli, «Poesia e musica», in Letteratura italiana, vol. VI, Teatro, musica, tradizione dei classici, Torino: Einaudi, 1986, p. 227-244, qui a p. 232. 
oralmente e non ne rimane pressoché alcuna traccia. D'altra parte era anche soggetta alle variazioni estemporanee proprie dell'improvvisazione; lo stesso di consueto succedeva per i testi che la accompagnavano, che in gran parte ne condivisero la tradizione non scritta.

Anche nella Summa artis rithimici vulgaris dictaminis di Antonio Da Tempo, il quale pure apparteneva agli ambienti in cui l'Ars nova era coltivata, il canto polifonico occupa solo una piccola sezione: è appunto lo specifico uso musicale a cui sono adibiti i madrigali.

Ma i trattatisti, come Dante o Francesco da Barberino, o appunto Da Tempo e l'anonimo autore del Capitulum de vocibus applicatis verbis, rimandano spesso alla musica e al canto anche per altri metri, riconducendo ad essa la struttura dei testi. È superfluo ripercorrere qui le dettagliate osservazioni di Dante sulle ragioni melodiche del metro della canzone, la cui struttura «si pertiene a li musici» (Conv., II, XI 9).

Nella trattazione di Antonio Da Tempo, il più vicino all'epoca e all'ambiente di Petrarca, pare corrispondere alla teoria del «divorzio» il fatto che egli parla di musica, oltre che per il madrigale, solamente per la ballata e per i rotondelli, forme metriche minori associate al canto corale ed al ballo. Mentre non fa alcun accenno ad una possibile veste musicale ad esempio per i sonetti, che infatti non risultano mai cantati nelle fonti medievali.

Da Tempo offre anche un ulteriore indizio a favore dell'estraneità dei sonetti al canto. Lassoluta mancanza di attestazioni di un uso musicale del sonetto fino al tardo Quattrocento, ${ }^{11}$ mentre sono frequenti le notizie per ballate e canzoni, contrasta curiosamente con la sua denominazione, poiché sonus nella terminologia medievale indica la melodia che riveste un testo: verosimilmente è questo il motivo per cui Da Tempo, che usa sonus in tale accezione, sente il bisogno di dedicare un breve capitolo del suo trattato alla questione Perché il sonetto ha questo nome, senza trovare un motivo plausibile. Come di consueto, le ragioni di tale dubbio sono date per scontate:

In secondo luogo, chiediamoci perché il sonetto si chiami così. Si può dire che il sonetto abbia questo nome perché, nella recitazione del componimento ["in rithimando» nel testo latino], suona bene alle orecchie di chi ascolta (ma questa spiegazione converrebbe di per sé a ciascun tipo di componimento). Oppure si può dire che nomi come questo furono inventati dagli antichi a loro discrezione e che delle loro etimologie non ci si deve curare troppo, tenuto conto dell'oggetto di cui questo trattato si occupa. ${ }^{12}$

11. Cfr. ad es. Agostino ZiIno, "Rime per musica e danza», in Enrico Malato (dir.), Storia della letteratura italiana, vol. II, Il Trecento, Roma: Salerno Editrice, 1995, IV. Verso un nuovo sistema di valori, sez. IV, cap. VII, p. 484.

12. Da Antonio DA TEMPO, Summa artis rithimici vulgaris dictaminis, cap. V, Quare dicatur sonetus. Da Tempo con sonus indica, come si diceva, la melodia che riveste il testo (cfr. ad. es. infra la citazione dal cap. VII) e la sua esecuzione vocale ( Et quantum ad sonum sive cantum, musici et cantores melius sciunt praedicta", cap. LI), ma nota che vulgariter esso è anche utilizzato ad indicare le ballate grandi e mezzane (cap. XXXVI-XXXVII). La ballata 
Ma se si esaminano altri luoghi della Summa si trovano affermazioni meno compatibili con il divorzio fra poesia e musica. Quando tratta delle canzoni, terzo metro preso in esame dopo sonetti e ballate, Da Tempo scrive:

In linea generale, per quanto riguarda la disposizione delle rime e il modo di sillabare, parlando sia dei versi che delle rime, possono essere composte con quelle forme e quelle rime con cui si compongono i sonetti e le ballate; e questo vale per ogni componimento volgare. ${ }^{13}$

Cioè stabilisce un'assoluta compatibilità nella modalità di composizione, ossia nei versi, nelle rime e nel modo di sillabare, non solo fra canzoni, sonetti e ballate: vi comprende anche gli altri metri minori, sicuramente sempre cantati ed ancillari alla musica. Lo stesso principio è esplicitato nel titolo di un capitolo, il VII: De forma soneti simplicis et scansione sillabarum cuiuslibet rithimi vulgaris. Dal punto di vista del rimatore si comporrebbero quindi nello stesso modo i rotondelli, che andavano cantati in coro e accompagnavano il ballo, ed i sonetti, che venivano recitati.

E quantomeno singolare che i trattati non registrino mai tale differenza, ossia accomunino esplicitamente i metri destinati alla recitazione con quelli per il canto, mentre la possibilità che un determinato testo non fosse destinato alla musica era ben chiara: ad esempio Da Tempo, riguardo ad uno specifico fatto prosodico, la sinalefe, scrive che essa va in particolare curata quando il testo è destinato ad essere cantato: «maxime in rithimis super quibus debet fieri sonus». ${ }^{14}$ Aggiungendo però che se questa regola non è rispettata il componimento non suona bene «secundum musicos et cantores»: ossia attribuisce la percezione di questa esigenza non all'autore del testo, ma a chi è incaricato di rivestirlo con una melodia.

Per meglio comprendere questo punto di vista, che mette programmaticamente sullo stesso piano la poesia colta ed i testi per musica, va notato che solo dal punto di vista moderno i metri per musica sono lontani da quelli propri della poesia. Infatti solo alla fine del ' 500 , in seguito all'evoluzione delle caratteristiche ritmiche della musica occidentale, si affermano nei testi ancillari alla melodia caratteristiche specifiche che li distanziano profondamente dai metri petrarcheschi: versi più brevi e ritmicamente più regolari, con uscite obbligatoriamente tronche, piane o sdrucciole a seconda della posizione del verso nella strofa. L'innovazione più caratteristica, che tuttora vale come indi-

è da lui intesa come destinata alla musica, e quindi non mostra alcuna perplessità su tale denominazione.

Il testo e la traduzione (provvisori) della Summa ci sono stati gentilmente forniti da Zeno Lorenzo Verlato, che ne sta preparando un'edizione tradotta.

13. «Et generaliter quantum ad rithimos et sillabicandi modum, sive loquamur de versibus sive de consonantiis, possunt compilari ex illis rithimis et consonantiis ex quibus compilantur soneti et ballatae; et sic de quolibet rithimo vulgari» (cap. XLVI, De cantionibus extensis et earum forma).

14. Dal cap. VII, De forma soneti simplicis et scansione sillabarum cuiuslibet rithimi vulgaris. 
zio principe per distinguere i metri concepiti per la musica, è la tendenza a concludere le strofe ed in generale le partizioni metriche con una parola tron$\mathrm{ca}$, cioè con una vocale accentata in corrispondenza della nota in tempo forte che tende a concludere i periodi e le frasi musicali a partire appunto dalle trasformazioni di fine ' 500 . Sono i metri delle arie del melodramma ma anche, più in generale, quelli che tuttora caratterizzano le canzonette, $i$ testi subordinati a una melodia. ${ }^{15}$

Diversa è la situazione ai tempi del Petrarca: i metri per musica, anche i più grossolani, erano ancora contigui e in maggior parte sovrapponibili a quelli della lirica. Ad esempio, sono brevi ballate di settenari ed endecasillabi, con rime piane, anche i rotondelli sopra citati. E se da un lato i rimatori, come si diceva, recitavano i loro testi, dall'altro le fonti dell'epoca testimoniano spesso come le loro composizioni fossero usualmente cantate, o meglio come fosse compito dei rimatori fornire i testi ai musicisti. Un noto esempio è la VII novella dell'ultima giornata del Decameron, dove una fanciulla, ammalatasi d'amore per Pietro d'Aragona, si confida con il «finissimo cantatore e sonatore» Minuccio d'Arezzo, con ogni probabilità un personaggio storico. ${ }^{16}$ Questi si reca allora dal rimatore Mico da Siena, «assai buon dicitore in rima a quei tempi», il quale fa sulla vicenda una ballata grande di tre strofe XY y X ABA$\mathrm{BAB} \mathrm{BC} \mathrm{X}$, che Boccaccio chiama però "canzonetta» e poi "canzone», in conformità all'uso corrente così come è spesso attestato dai trattatisti. ${ }^{17} \mathrm{Il}$ musico Minuccio impara tali versi e poi li fornisce "d'un suono soave e pietoso» per cantarli con la viola di fronte al re.

Come è noto, anche nell'accezione originaria di Roncaglia il divorzio fra musica e poesia va inteso come una separazione dei ruoli: «la grande maggioranza dei poeti aulici italiani componevano solo testi verbali, lasciando un loro eventuale (non obbligatorio) rivestimento melodico a musici professionisti». ${ }^{18}$

Che poi alcuni testi, in particolare i più umili, fossero scritti espressamente per una melodia esistente, trova conferma ad esempio in una singolare annotazione evidenziata da Pirrotta nell'anonimo Capitulum de vocibus applicatis verbis: le «ballade», ossia le ballate più semplici e popolari, finalizzate alla danza, «sunt verba applicata sonis», mentre $\mathrm{i}$ «soni sive sonetti», cioè in questa particolare terminologia le ballate più ampie, che come nell'esempio da Boccaccio venivano cantate senza danza ed erano affini alle canzoni, "sunt verba appli-

15. Una visione d'insieme della questione è in Luca ZULIANI, «Sull'origine delle innovazioni metriche di Gabriello Chiabrera", Stilistica e metrica, 3, 2003, p. 91-128.

16. Cfr. Stefano Carrai, «Un musico del tardo Duecento (Mino d'Arezzo) in Nicolò de' Rossi e nel Boccaccio», Studi sul Boccaccio, XII, 1980, p. 39-46.

17. Cfr. ad es. Antonio da Tempo, Summa, cap. XXXV De ballatis: «Largo enim modo sumpto vocabulo, cuiuscunque generis ballatae, de quibus infra dicetur, possunt appellari et vulgariter appellantur cantiones»; e poi nel cap. XLVI: «ballatae etiam cantiones vulgariter appellantur».

18. Aurelio Roncaglia, "Sul "divorzio fra musica e poesia” nel Duecento italiano», in L’Ars Nova italiana del Trecento, IV, atti del $3^{\circ}$ Congresso internazionale sul tema «La musica al tempo del Boccaccio e i suoi rapporti con la letteratura» (Siena-Certaldo 19-22 luglio 1975), Certaldo: Edizioni Centro di Studi sull'Ars Nova italiana del Trecento, 1978, p. 390. 
cata solum uni sono». Ossia, mentre le prime si possono adattare a molte delle melodie correnti, le seconde richiedono una sola melodia espressamente composta per la loro particolare struttura metrica. ${ }^{19}$

$\grave{E}$ anche spesso attestato che i testi di più ampio respiro, ossia innanzitutto le grandi canzoni, non erano destinati a essere musicati. Basti citare la celebre chiosa ad Amor che ne la mente mi ragiona cantato da Casella (Purg. II 112) nel commento dantesco dell'Anonimo Fiorentino: «È vero che, per che le canzoni morali, come fu questa, non suole essere usanza di intonarle, credo che questa Amor che ne la mente mi ragiona fosse principio di qualche ballata, o suono". ${ }^{20}$

Ma se da un lato i rimatori italiani, a differenza di quanto accadeva per i trovatori, possono comporre versi anche a prescindere da un loro uso musicale, dall'altro comunque continuano a farlo su metri che sono concepiti per la musica, nella tipologia dei versi come nella struttura delle strofe. Da un lato appare evidente dalle fonti che, almeno a partire dal ' 300 , vengono musicate e cantate in particolare le ballate, che spesso sono eseguite da una sola voce e senza danza, come le canzoni nella sistemazione dantesca: ${ }^{21}$ difatti canzoni sono correntemente chiamate, come si è accennato. Ma dall'altro è palese che nella coscienza dei contemporanei una stanza di ballata e una stanza di canzone hanno identica struttura: vi è solo una differenza di dimensioni, come nota $\mathrm{Da}$ Tempo; ${ }^{22}$ e anche un sonetto per i trattatisti è diviso allo stesso modo in piedi e volte.

La preferenza per la forma «ballata di settenari ed endecasillabi» nei testi per musica permane anche nel ' 400 . Per tale secolo è possibile individuarla tramite una fonte particolarmente rappresentativa dell' uso musicale corrente: i laudari quattrocenteschi contengono testi religiosi ed edificanti applicati a melodie profane in voga. Ogni testo è preceduto dall'indicazione "cantasi come», seguita dal primo verso della canzone originale. Spesso di tale originale, dato come noto nel laudario, non resta altra traccia, ma la sua forma metrica è ricostruibile attraverso il contrafactum della lauda: ancora a questa altezza buona parte dei testi è costituita appunto da ballate di settenari ed endecasillabi, sia pure con frequenti anisosillabismi. Lo stesso metro è del resto bene attestato anche nella produzione, tutta per musica e per altri versi metricamente innovativa, di Leonardo Giustinian. ${ }^{23}$

19. Cfr. Nino PirrotTa, «Ballate e "soni” secondo un grammatico del Trecento», in ID., Musica tra Medioevo e Rinascimento, Torino: Einaudi, 1984.

20. Dal Commento alla Divina Commedia d'Anonimo Fiorentino del secolo XIV, a cura di Pietro FANFANI, Bologna: Romagnoli, 1866-74. Quanto all'uso del termine suono, anche Da Tempo nota che esso è vulgariter usato per le ballate grandi e mezzane: cfr. supra la nota alla citazione dal cap. V della Summa, e anche i «soni sive sonetti» nel senso di ballate nell'appena citato Capitulum de vocibus applicatis verbis.

21. Cfr. ad es. Agostino ZIINO, «Rime per musica e danza» cit., p. 476-477.

22. "Sunt tamen breviores ballatae superiores in verbis quam cantiones extensae, ut infra patebit», Summa, cap. XLVI, De cantionibus extensis et earum forma.

23. Cfr. Luca Zuliani, «Sull'origine delle innovazioni metriche di Gabriello Chiabrera» cit., p. 95 e passim. 
Tornando al '300, va poi considerato che l'esecuzione del testo, fosse recitato o cantato, aveva un'importanza incommensurabilmente maggiore rispetto a quanto accade oggi, e non solo a causa della maggiore abitudine alla memorizzazione. La stessa lettura personale era di consueto attuata articolando a voce il testo, e le rime volgari venivano correntemente recitate non solo dai loro autori, ma anche da altri che oltre a comporre raccoglievano e recitavano testi altrui: il volgare et vulgatum genus descritto da Petrarca al Boccaccio nella lettera sopra citata. Oltretutto di consueto i destinatari di elevata posizione sociale, come le egregie domine sopra citate da Donato degli Albanzani o il re Pietro della novella di Boccaccio, non vedevano pressoché mai un testo scritto: c'era sempre qualcuno che leggeva o scriveva per loro. ${ }^{24}$

C'è un ulteriore e finale elemento che, inserito in questo quadro, aiuta a definirne i tratti: proviamo a supporre per i testi volgari una recitazione fortemente cantilenata in base alla struttura metrica, come nella poesia popolare o come, ad esempio, tuttora succede nella recitazione dei metri canonici della poesia inglese; e consideriamo che dall'altra parte c'è un'esecuzione musicale che, affidandosi principalmente alla voce, si conforma rigorosamente al metro ${ }^{25}$ e non è legata ad una struttura ritmica nel senso moderno, ma è simile piuttosto alla tecnica del recitativo del melodramma, o alla "parola cantata» della musica gregoriana. E che oltretutto, non essendo scritta, poteva seguire moduli stereotipati e nello stesso tempo essere soggetta alla variabilità dell'improvvisazione. In un simile contesto, come scrive Petrobelli,

la musica viene insomma concepita come semplice intensificazione, al puro livello sonoro, della parola; essa ha principalmente la funzione di estrinsecarne l'articolazione formale, il suo disporsi nel tempo. ${ }^{26}$

Il presente intervento ha appunto preso le mosse da uno studio sui rapporti fra sintassi e metro nei Rerum vulgarium fragmenta e, più in generale, nella lirica italiana fra le origini e il ' 500 . In esso si è ipotizzato che i metri della poesia italiana, dalle origini fino ancora all'altezza di Petrarca, implicassero una precisa e rigida struttura ritmico-intonativa nell'esecuzione della poesia, sulla base dei versi e delle altre partizioni metriche. La sintassi poteva adattarsi a tale struttura, oppure sfruttarla ponendosi in voluto contrasto con essa, ma non poteva ignorarla. ${ }^{27}$

Per spiegare strutture di questo tipo, rintracciate tramite una schedatura analitica della sintassi dei testi, si è ipotizzata un tipo di recitazione fortemente cantilenata e ritmata in base al metro. Ma questo tipo di struttura rit-

24. Per l'intera questione cfr. Paul SAENGER, «Leggere nel tardo Medioevo», in Guglielmo CAVAllo e Roger CHARTiER (a cura di), Storia della lettura, Roma-Bari: Laterza, 1995, p. 117154.

25. Cfr. ad es. infra nelle note la citazione da Pierluigi Petrobelli, «Poesia e musica» cit., p. 235.

26. Pierluigi Petrobelli, «Poesia e musica» cit., p. 236.

27. cfr. Luca Zuliani, «Sintassi e metro nei Rerum vulgarium fragmenta», Critica del testo, VI, 1, 2003, p. 454-498. 
mico-sintattica è propria anche di un testo musicato in base alla sua forma metrica, un testo cioè in cui la struttura della melodia coincide con quella del metro.

Nella situazione qui descritta, in cui la poesia è quasi sempre eseguita e fruita a voce e in cui recitazione e canto, pur rimanendo ben distinti, si svolgono secondo modalità affini, la distanza fra versi e musica è molto più ridotta di quanto potrebbe pensare un moderno. Mantengono una contiguità con la musica testi che sarebbe assai impegnativo rivestire con una melodia, come le canzoni di architettura più ambiziosa. Esse infatti sono comunque composte su una struttura metrica concepita per una veste musicale e la sintassi del testo tiene presente la struttura ritmica sottesa al metro. Vengono solo recitate, ma in un modo che corrisponde ad una linea melodica "di grado zero».

Lo stesso vale, dall'altro lato, per i sonetti. Un esempio fra tanti: quando Francesco Sacchetti invia una ballata al musico Francesco degli Organi, accompagnandola come spesso accade col sonetto Veggendo tante piaghe e tanti segni in cui lo prega di musicarla, e il musicista esaudisce la richiesta rimandandogli il testo intonato e un sonetto di risposta ${ }^{28}$ non c'è ragione di supporre differenze sostanziali, nella composizione come nella recitazione, fra la ballata e i sonetti, anche se questi non riceveranno una melodia.

Il sonetto sarebbe quindi concepito come una sorta di «modulo metrico di base»: può servire per mandare un messaggio a margine di un altro testo o per uno scambio di opinioni, ma resta in qualche modo un genere interno ai rimatori, escluso programmaticamente da eventuali usi musicali, per i quali è invece richiesto un testo metricamente mosso che la nuova melodia può rivestire come «intensificazione della parola». Una simile situazione può forse servire a capire un punto assai discusso del De vulgari eloquentia: come mai per Dante fosse tanto ovvio che il sonetto fosse una forma metrica inferiore alla ballata e alla canzone. ${ }^{29}$

Ma torniamo finalmente a Petrarca: in che rapporti sono le sue rime volgari con il quadro che abbiamo disegnato? Vi è un luogo in cui egli tocca i legami con la pratica musicale del suo tempo: è la familiare X, 3 al fratello Gherardo, in cui Petrarca, approvando la scelta di farsi monaco, rievoca la loro giovinezza perduta in preoccupazioni mondane: ricorda diffusamente come entrambi ponessero un'attenzione esagerata al proprio aspetto, soffermandosi in particolare sulle faticose acconciature dei capelli, ottenute tramite dolorosi calamistri da indossare di notte, che finivano per lasciarli con la capigliatura ben modellata ma con la fronte segnata e dolente. E infine ricorda

28. Cfr. Franco Alberto Gallo, Dal Duecento al Quattrocento, in Letteratura italiana (Einaudi), vol. VI, Teatro, musica, tradizione dei classici, cit., p. 257.

29. De vulgari eloquentia, II, III, 5: «ergo cantiones nobiliores ballatis esse sequitur extimandas, et per consequens nobilissimum aliorum esse modum illarum, cum nemo dubitet quin ballate sonitus nobilitate excellant». Un'analoga gerarchia è anche in Francesco DA BARBERINO, I Documenti d'Amore, a cura di Francesco Egidi, Roma: Società Filologica Romana, 1905-1927, vol. II, p. 262. 
quanta cura e quanto tempo spendemmo per rendere nota al pubblico la nostra frenesia e divenire favola alle genti. Quante volte contorcemmo le sillabe, quante volte spostammo le parole; ed infine cosa non facemmo perché quel nostro amore che, se non era possibile estinguere, almeno doveva essere tenuto celato dal pudore, venisse invece cantato in versi destinati ad attirare applausi [denique quid non fecimus ut amor ille, quem si extinguere non erat, at saltem tegi verecundia iubebat, plausibiliter caneretur?]. Eravamo elogiati per i nostri studi e l'olio del peccatore ungeva il capo di noi che deliravamo. ${ }^{30}$

L'amore di cui parla fu, spiega Petrarca, quello verso Laura, mentre il fratello amò un'altra fanciulla anch'ella morta giovane. Le parole con cui Petrarca deplora i versi ispirati a questi amori ritornano uguali in celebri luoghi del Canzoniere: «divenire favola alle genti» («multorum [...] populorum fabula») rimanda ovviamente a «ma ben veggio or sì come al popol tutto | favola fui gran tempo", nella prima terzina del sonetto proemiale, che fu probabilmente scritto nello stesso anno di questa lettera o nel successivo; ${ }^{31}$ mentre ad esempio «quanti sospiri, quanti lamenti, quante lacrime abbiamo sparso al vento!» poco più sotto ("quot suspiria quot lamenta quot lacrimas in ventos effudimus!») riecheggia "quante lagrime, lasso, et quanti versi | ò già sparti al mio tempo", $R V F$ 239, 13-14, o anche "Vergine, quante lagrime ò già sparte, | quante lusinghe et quanti preghi indarno, | pur per mia pena et per mio grave danno!», nella canzone conclusiva alla Vergine, ai v. 79-81.

Petrarca conclude così la rievocazione a Gherardo:

E ora dunque dimmi, tu che da nemico sei divenuto amico di Dio, e da avversario suo cittadino, tu che ormai hai respinto l'antico e abbracciato il nuovo, dimmi: cosa hanno di comune quelle nostre vacue canzonette [cantiuncule inanes] piene di turpi e false lodi di donna, di un'invereconda ed aperta confessione di libidine; che hanno di comune con le vostre lodi divine e le sacre veglie $[\ldots]$ ?

Ora, per quanto siano sempre riduttivi i pochissimi accenni che Petrarca fa delle sue nugae volgari, è difficile pensare, quando parla di cantiuncule inanes condivise col fratello nell'ambiente mondano avignonese, ch'egli alluda alle poesie che oggi leggiamo nei Rerum vulgarium fragmenta, qualunque forma esse avessero a questa altezza, ossia probabilmente nel '49. Oltretutto nei tempi che qui rievoca Petrarca si trovava in Provenza, quindi c'è persino la possibilità che almeno in parte fossero versi in lingua d'oc. Si tratta però delle sue prime rime per Laura: Petrarca pare scrivere ch'esse erano destinate al canto, probabilmente da parte di altri, anche perché egli descrive solo la composizione del testo, tacendo della melodia. Che il verbo canere sia usato in senso letterale,

30. Da F. Petrarca, Epistole cit., p. 258-286, e così le citazioni successive dalla stessa lettera.

31. La lettera è con ogni probabilità del 1349, il sonetto del '49-'50. Cfr. Francisco Rico, "Rime sparse", "Rerum vulgarium fragmenta". Para el titulo y el primer soneto del "Canzoniere" ", Medioevo romanzo, 3, 1976, p. 101-138 e Arnaldo FORESTI, Aneddoti della vita di F. Petrarca, Brescia: Vannini, 1928, p. 98-105. 
in riferimento ad una fase successiva alla composizione, è suggerito dal contesto, tutt'altro che aulico («ed infine cosa non facemmo perché quel nostro amore $[. .$.$] venisse invece cantato in versi destinati ad attirare applausi») e dal$ termine cantiuncule poco più sotto, ${ }^{32}$ studiatamente contrapposto con i canti in lode di Dio (divine laudes) di cui era composta la liturgia monastica.

Quello descritto non è altro che il procedimento usuale: il rimatore affida a un musico i propri testi perché siano rivestiti ed eseguiti. Abbiamo visto anche come tali rime per musica avessero metri analoghi o contigui a quelli del Canzoniere: è quindi anche possibile che questo ne conservi alcune rielaborazioni. Invece è del tutto normale che non resti traccia alcuna del loro uso e della loro veste musicale, che, come si è visto, al di fuori dell'Ars nova non avevano una tradizione scritta.

Che cosa risulta infine da questa ricostruzione? Innanzitutto che, ai tempi di Petrarca, musico e poeta sono due figure nettamente distinte, ma è prematuro parlare di «divorzio» fra musica e poesia: la composizione poetica e quella musicale continuano a svolgersi affiancate e le forme della poesia hanno, nella percezione collettiva come nella prassi dell'esecuzione, una ragione musicale. E un legame che ormai tende a indebolirsi per i testi della fascia più alta, ma d'altro canto rimane solido e continuo per quelli più corsivi. La svolta, il distacco vanno allora posticipati al ' 500 maturo, quando l'unica possibile veste melodica di una poesia lirica è il madrigale nell' accezione musicale e cinquecentesca del termine: una melodia che si ritaglia nel testo una struttura ritmica propria e indipendente dalle partizioni metriche, seguendo la sintassi che pure, nei testi rinascimentali, prende forme incompatibili con le pause indicate dal metro. $\mathrm{Ne}$ risulta una costante asincronia tra frasi musicali e versi, che in origine erano la stessa cosa e sempre coincidono nella musica medievale: ${ }^{33}$ è appunto il segno dell'avvenuto divorzio fra le forme della poesia e le forme della musica.

$\mathrm{Vi}$ è poi una questione limitata al nostro autore. Le fonti coeve, da Boccaccio a Villani a Salutati, sono concordi nel riportare che Petrarca amava cantare e suonare il liuto: «lira mire cecinit, unde labores studii modeste levabat», 34 scrive Villani che, come altri, si dilunga anche a ricordare la soavità della sua voce. Per quanto presente anche in autori che di Petrarca furono amici inti$\mathrm{mi}$, questa notizia potrebbe non avere fondamento o essere un' esagerazione, considerando che il diretto interessato non fa mai il minimo accenno ad una sua pratica musicale. Ma, come è noto, nel suo testamento Petrarca si premu-

32. Cantiuncula è un hapax nelle prose di Petrarca (che non usa mai neppure il poco classico cantio) ed è un calco sull'usuale canzonetta, che indica appunto i testi ancillari ad una melodia.

33. Petrobelli evidenzia l'assenza di asimmetrie di questo tipo come tratto distintivo della musica medievale: "L'articolazione del fatto sonoro (sia esso monodico, cioè affidato ad una sola voce, oppure affidato a più voci) segue la disposizione del testo poetico secondo i versi, le strofe, e le ripetizioni dei versi (nei ritornelli e nelle "volte"), ad un punto tale per cui la disposizione del testo musicale si atteggia secondo quella della poesia, anzi da essa dipende» (Petrobelli, Poesia e musica cit., p. 235).

34. Filippo Vellani De origine civitatis Florentie et de eiusdem famosis civibus, edidit Giuliano Tanturli, Padova: Antenore, 1997, p. 92. 
ra di lasciare il «leutum meum bonum», il migliore dei suoi liuti, all'amico Tommaso Bambasio (che probabilmente non era nemmeno lui un musicista) ${ }^{35}$ perché lo suoni «non pro vanitate seculi fugacis, sed ad laudem Dei eterni». Il liuto si usava per accompagnarsi nel canto: la musica solo strumentale a questa altezza aveva un ruolo del tutto secondario e di consueto ancillare alla danza. La notizia delle pratiche canore di Petrarca va quindi ritenuta esatta.

Una probabile conferma indiretta arriva dal fatto che Petrarca, nelle sue prose, ricorda spesso l'esempio di personaggi celebri dell'antichità che si diedero alla studio e alla pratica della musica, anche in tarda età. Un ampio elenco, che include anche esempi negativi, è nel capitolo dedicato alla dolcezza della musica nel De remediis utriusque fortune, I 23, 16. È la risposta della ragione all'affermazione "delectat canere», "cantare procura gioia»:

La Grecia si dilettò di ciò, ora anche voi. Presso di loro, quando non si sapeva né cantare né suonare uno strumento, si era considerati rozzi. Cicerone racconta che ciò accadde a Temistocle, l'ateniese fra i greci illustrissimo, per aver rifiutato di suonare una lira durante un banchetto; e che Epaminonda tebano cantava splendidamente con il liuto, forse appunto per evitare l'infamia di cui s'è detto. Si dice pure che Socrate nella sua vecchiaia si mise a studiare la lira, e ciò è davvero degno di nota. Quindi non c'è da stupirsi che Alcibiade fosse destinato da suo zio Pericle allo studio del flauto, occupazione molto prestigiosa fra i greci, perché facesse esperienza anche di questa fra le arti liberali [...].

Presso di voi questa passione per la musica non è giunta ad occupare lo spirito di tutti i vostri principi, ma ha occupato in particolare gli animi peggiori: Caligola si appassionò massimamente del canto e del ballo, ed è incredibile a dirsi quanta passione aveva Nerone per la cetra e per il suono della sua voce. [...] E ci sarebbero altri esempi.

Per quanto fosse stata messa in guardia, anche l'epoca in cui voi siete si è data al piacere della musica. E dilettarsene castamente e sobriamente implica una sorta di umanità, mentre è grande vanità lasciarsene catturare e abbandonarvisi mollemente. ${ }^{36}$

In queste frasi come nei suoi silenzi sull'argomento, è possibile indovinare la discrezione con cui Petrarca tralasciava di palesare questa sua occupazione mondana. Va anche considerato che la separazione fra i ruoli di poeta e di musico aveva ovviamente abbassato il prestigio sociale di cantatori e sonatori.

Ma che cosa cantava Petrarca? Come è chiaro anche dalla raccomandazione testamentaria all'amico Bambasio, si trattava almeno inizialmente di canti profani, quindi solitamente in volgare.

35. L'opinione vulgata che Bambasio fosse un musicista è stata recentemente confutata da Stefano Campagnolo al congresso Petrarca in musica, Arezzo, 18-20 Marzo 2004 (atti in corso di stampa).

36. Traduzione di servizio da PÉTRARQue, Les remèdes aux deux fortunes, Texte établi et traduit par Christophe CARRAUD, Grenoble: Jérôme Million, 2002, vol. 1, p. 122. Gli aneddoti qui riuniti ricorrono spesso isolati altrove. Ad esempio, la tardiva passione di Socrate per la lira è ricordata anche nella Familiare XVII, 8, 5, in due Senili (I, 5, 16 e XVI, 6) e in Rerum memorandarum libri I, 9, 1. 
Difficile che fra questi usasse cantare i rotondelli o le ballate più semplici, associati alla danza e all'esecuzione corale. Neppure poteva cantare i canti popolari che talvolta si trovano menzionati nelle fonti, spesso in bocca a personaggi di umile estrazione. ${ }^{37}$ E piuttosto improbabile anche che lasciando da parte il liuto usasse intonare, insieme con i suoi amici arsnovisti, musica polifonica. Poteva, forse, cantare talvolta una sola delle voci di un madrigale, eseguendo l'altra con il liuto: di questa possibilità abbiamo attestazioni. ${ }^{38}$

Ma con ogni probabilità Petrarca eseguiva innanzitutto ballate e canzoni. Certo non si può sapere se usasse cantare le proprie e se fosse mai lui a dare il sonus, la melodia. Ma di sicuro tali rime, se non erano quelle del Canzoniere, erano metricamente e stilisticamente compatibili con esse.

La menzione del liuto e le notizie dei contemporanei sui passatempi musicali del Petrarca hanno già portato un tempo ad illazioni un po' ingenue, frutto della temperie romantica: il Foscolo ad esempio scrisse che «il Petrarca compose i suoi versi al suono del suo liuto, che legò nel testamento ad un amico; ed ebbe voce dolce, flessibile e di grande estensione». ${ }^{39}$ Come si è visto, la composizione delle rime volgari in Petrarca e nei suoi contemporanei risulta invece slegata da una specifica veste musicale e programmaticamente precedente ad essa.

Ma d'altro canto il legame con la melodia resta solido e del tutto difforme dalla concezione moderna. Ciò che maggiormente fa la differenza è la presenza di una tradizione, di una pratica costante e diffusa che precede le sistemazioni teoriche (si veda Da Tempo, quando si vanta d'avere per la prima volta trovato un nome a usuali forme delle rime volgari ${ }^{40}$ e comporta, oltre a determinate strutture metriche, anche specifiche modalità di composizione ed esecuzione.

Bisogna però concludere ricordando che Petrarca fa parte di questa tradizione, ma nel contempo è anche volto al suo superamento, tanto da indicare la strada alle generazioni successive. Egli raccolse e rielaborò le sue liriche staccandole completamente dal contesto in cui erano nate: la sua intenzione, quando prende forma il Canzoniere, non è più cantare le lodi della donna amata in un contesto mondano, ma bensì tracciare il percorso esemplare di un'anima. Il pubblico sono gli scelti amici e corrispondenti della maturità e, ovviamente, i posteri; non più il profanum vulgus dove correvano, recitate e cantate, le rime volgari.

37. Cfr. ad es. F.A. GAllo, «Dal Duecento al Quattrocento» cit., p. 248.

38. Cfr. ad es. Bianca BECHERINI, «L'Ars Nova italiana del Trecento. Strumenti ed espressione musicale», in L'Ars Nova italiana del Trecento, I, atti del Primo Convegno Internazionale (Certaldo 23-26 luglio 1959), Certaldo: Edizioni Centro di Studi sull'Ars Nova italiana del Trecento, 1962, p. 40-56.

39. Da Ugo Foscolo, Saggio sopra la poesia del Petrarca, in Opere, vol. X, Saggi e discorsi critici (1821-1826), a cura di C. Foligno, Firenze: Le Monnier, 1953, p. 251. Più recenti ma appena più sfumate sono le affermazioni di Dante BIANCHI nel saggio «Di alcuni caratteri della verseggiatura petrarchesca», Studi petrarcheschi, 6 (1956), p. 81-121 (p. 82).

40. Summa, cap. LVIII: «E io stesso, in questo manuale, ho posto non pochi nomi a determinati componimenti, curando che essi si adattassero alla loro effettiva realtà» («Et plura nomina ego in hac arte certis rithimis imposui rebus consequentia»). 